\title{
ESTUDO DE CORRESPONDÊNCIA DE ELEMENTOS METADADOS: DUBLIN CORE E MARC 21
}

Maria das Dores Rosa Alves

Marcia Izabel Fugisawa Souza

\section{Resumo}

A tecnologia da informação tem possibilitado o desenvolvimento de aplicações e metodologias de tratamento descritivo, como é o caso dos padrões de metadados, permitindo novas abordagens práticas para a organização da informação, principalmente em meio eletrônico. A adoção de padrão de metadados propicia a interoperabilidade entre aplicações e o compartilhamento de dados entre sistemas. Baseado nesse pressuposto, foi realizado um estudo da correspondência entre o padrão Dublin Core e o Formato MARC 21, visando fornecer subsídios para o desenvolvimento de ferramentas de conversão de dados oriundos da catalogação. A maximização de esforços na integração e intercâmbio de dados, bem como a sistematização das relações entre esses padrões, propiciam o reuso de conjuntos de metadados na mesma organização.

\section{Palavras-chave}

Dublin Core; Formato MARC 21; Metadados; Interoperabilidade; Catalogação de recursos eletrônicos; Agência de informação Embrapa

\section{STUDY OF THE CORRESPONDENCE OF METADATA ELEMENTS : DUBLIN CORE AND MARC 21}

\begin{abstract}
The information technology has been making possible the development of applications and methodologies of material descriptive treatment, as it is the case of the metadata standards, allowing new practical approaches for the organization of the information, mainly in electronic means. The adoption of metadata standard propitiates the interoperability between applications and sharing of data among systems. Based on that presupposition, a study of the correspondence was accomplished between the Dublin Core standard and the MARC 21 format, seeking to supply subsidies for the development of data conversion tools originating from of the cataloguing. The maximization of efforts in the integration and exchange of data, as well as the systemization of the relationships among those standards propitiate the reuse of metadata sets in the same organization.
\end{abstract}

\section{Keyword}

Dublin Core; MARC 21 format; Metadata; Interoperability; Cataloguing of electronic resources; Embrapa's information agency 


\section{INTRODUÇÃO}

O valor da informação aumenta com o seu uso e quando combinada com outra informação. A informação é mais útil, certamente, quando ela pode ser comparada, combinada e compartilhada com outra informação. Com base no pressuposto de que o sucesso no compartilhamento de recursos e aplicações requer o estabelecimento de padrões de tratamento de dados e informações (COMITÊ... 2005), é que se priorizou este estudo de correspondência entre o formato MARC 21 e o Dublin Core.

A adoção de padrões, normas e modelos internacionais, fruto de esforços coletivos na área de representação bibliográfica é essencial para o intercâmbio de informações. O desenvolvimento constante das tecnologias da informação e de comunicação tem possibilitado o avanço metodológico, como é o caso dos modelos metadados, que propiciam novas práticas para a organização e tratamento da informação digital, proporcionando diferentes mecanismos de busca e recuperação.

O objetivo deste estudo é sistematizar a relação existente entre os dois padrões, visando subsidiar um possível reaproveitamento de catalogações de outros sistemas dentro da própria empresa, tornando possível expandir os repositórios digitais de informação e racionalizar o trabalho do bibliotecário catalogador, propiciando, ainda, economia de recursos.

A Empresa Brasileira de Pesquisa Agropecuária (Embrapa) desenvolveu a Agência de Informação Embrapa, um sistema de informação tecnológica voltado aos interesses do agronegócio, disponível na internet, para acesso instantâneo e na íntegra a recursos eletrônicos. Além de outros benefícios, a Agência de Informação Embrapa dedica-se a agregar valor à informação e ao conhecimento, antes de disponibilizá-los, para isso adota padrões de tratamento da informação compatíveis com o ambiente web, o que possibilita a interoperabilidade com outros sistemas e mecanismos de busca. 
A Agência utiliza o padrão Dublin Core (DC) para o tratamento descritivo dos recursos de informação. A indexação e a categorização de assuntos é baseada no uso de tesauros e vocabulários controlados, o que propicia a geração de metadados com qualidade. Para Souza et al. (2005), o DC provê uma base para interoperabilidade semântica entre outros formatos mais complexos já consolidados na forma de padrões internacionais de descrição de informação eletrônica, no caso aqui tratado e objeto deste estudo o formato MARC 21.

\section{METADADOS}

A utilização de metadados na organização eletrônica de recursos, vem ao encontro da necessidade crescente de descobrir e disponibilizar informações na internet e nas intranets. O uso de padrões de metadados possibilita aos sistemas de informação e de gestão do conhecimento a integração e o compartilhamento de recursos e aplicações.

De uma forma mais simples, pode-se definir metadados como dados codificados e estruturados que descrevem a característica de recursos de informação, sejam eles produtos ou serviços. Elementos como autor, título, assunto são exemplos de metadados e podem ser usados para descrever tanto um livro em um catálogo de uma biblioteca on line ou não, quanto para descrever uma home page, uma base de dados ou qualquer outro recurso eletrônico em ambiente web (SOUZA et al., 2000).

Os elementos de metadados têm o propósito primário de descrever, identificar e definir um recurso de informação com o objetivo de modelar e filtrar o acesso. Os metadados são importantes na organização, gestão e recuperação da informação digital, principalmente. Nesse sentido, são adotados procedimentos técnicos de catalogação, indexação e categorização dos conteúdos informacionais, o que possibilita a integração de fontes diversificadas e heterogêneas de informação. 
Assim, os metadados enriquecem ou complementam os objetos ou serviços referenciados, aumentando o potencial informativo. Portanto, são dados definidores que fornecem informação sobre ou documentação de outros dados dentro de uma aplicação ou de um ambiente. Podem, ainda, conter informação descritiva sobre o contexto, a qualidade e a condição ou características dos dados (RELVÃO, 2003).

\section{INTEROPERABILIDADE}

Os metadados são importantes na descoberta de recursos, ou seja, possibilitam a busca de recursos de informação por critérios relevantes, identificação de recursos, agrupamento de recursos similares, diferenciação de recursos não similares e a obtenção de informação de localização (GONÇALVES, 2005). A descrição de recursos por meio de metadados permite que eles sejam compreendidos por programas, sendo pois, essenciais à interoperabilidade entre aplicações e ao compartilhamento de dados entre sistemas.

A capacidade de bases de dados trocarem e compartilharem documentos, consultas e serviços, usando diferentes plataformas de hardware e software, estrutura de dados e interfaces, é chamada de interoperabilidade. Através dessa troca e compartilhamento são realizadas interações entre sistemas. Porém, as informações devem estar organizadas eficientemente para que essas interações aconteçam. E a principal característica para o sucesso dessas interações é a consistência, a qual é alcançada através do uso de padrões.

O objetivo da interoperabilidade é disponibilizar serviços coerentes aos usuários, a partir de componentes tecnicamente diferentes e gerenciados por organizações diferentes (CHAVES, 2002). Isto requer cooperação em três níveis: técnico, de conteúdo e organizacional. No nível técnico se incluem os formatos, protocolos, sistemas de segurança, etc., para que as mensagens possam ser trocadas; no nível de conteúdos estão os dados e metadados, incluindo os protocolos semânticos, usados para a interpretação da informação; no nível organizacional se enquadram as regras básicas para acesso, preservação de coleções e serviços, pagamentos, autenticação, etc. 
No âmbito deste estudo são considerados o nível técnico, nos aspectos que cobrem o formato, e o nível de conteúdo de dados e metadados para troca de informações entre sistemas.

\section{DUBLIN CORE}

O Dublin Core é um padrão de metadados, composto por 15 elementos, planejado para facilitar a descrição de recursos eletrônicos. Souza et al. (2000) destaca que as principais características deste padrão são a simplicidade na descrição dos recursos, entendimento semântico universal (dos elementos), escopo internacional e extensibilidade, o que permite a adaptação às necessidades adicionais de descrição.

Dublin Core vem sendo adotado internacionalmente por diversas instituições de renome. No Brasil, a exemplo do que vem ocorrendo em muitos países, instituições envolvidas na geração, recuperação e uso de recursos eletrônicos, como é o caso da Embrapa, vem sentindo a crescente necessidade de estabelecimento de padrões de descrição de conteúdo de recursos de informação e a geração de metadados (SOUZA et al., 2005).

O DC pode ser inserido em uma página HTML (Hypertext Markup Language) e utiliza a linguagem XML (eXtensible Markup Language). Adota a sintaxe do RDF (Resource Description Framework), possui um conjunto de 15 elementos básicos (DUBLIN CORE METADATA INITIATIVE, 2004), apresentados no Quadro 1, que podem ser implementados livremente para atender as necessidades de cada usuário, e, ainda é um formato padrão adotado para efetuar a interoperabilidade entre outros formatos.

Os documentos no padrão DC são considerados como objetos e integram todos os possíveis tipos de materiais e seu tratamento técnico, sejam bases de dados, imagens digitais, banco de imagens, textos eletrônicos, vídeos e filmes em formato digital e não- 
digital, objeto multimídia, gravações sonoras em formato digital e não-digital (XAVIER, 2005).

\begin{tabular}{l|l} 
Elementos & Descrição \\
Título & Nome dado ao recurso \\
$\begin{array}{l}\text { Criador } \\
\text { Assunto }\end{array}$ & $\begin{array}{l}\text { Tema do conteúdo do recurso. Pode ser expresso em palavras-chaves e/ou } \\
\text { Categoria. Recomenda-se o uso de vocabulários controlados }\end{array}$ \\
\hline Descrição & Relato do conteúdo do recurso. Exemplos: texto livre, sumário e resumo \\
\hline Publicador & Entidade responsável por tornar o recurso disponível \\
\hline Colaborador & Entidade responsável pela contribuição intelectual ao conteúdo do recurso \\
\hline Data & Data associada a um evento ou ciclo de vida do recurso \\
\hline Tipo & $\begin{array}{l}\text { Natureza ou gênero do conteúdo do recurso. Exemplos: texto, imagem, som, } \\
\text { dados, software }\end{array}$ \\
\hline Formato & Manifestação física ou digital do recurso. Exemplos: html, pdf, ppt, gif, xls \\
\hline Identificador & Referência não-ambígüa (localizador) para o recurso dentro de dado contexto \\
\hline Fonte & Referência a um recurso do qual o presente é derivado \\
\hline Idioma & Língua do conteúdo intelectual do recurso \\
\hline Relação & Referência para um recurso relacionado \\
Cobertura & Extensão ou escopo do conteúdo do recurso; pode ser temporal e espacial \\
Direitos autorais & Informação sobre os direitos assegurados dentro e sobre o recurso \\
\hline
\end{tabular}

Quadro 1 - Elementos metadados Dublin Core.

\section{FORMATO MARC 21}

MARC é um acrônimo de MAchine-Readable Cataloging: um conjunto de padrões para identificar, armazenar, e comunicar informações bibliográficas em formato legível por máquina, de forma que diferentes computadores e programas possam reconhecer, processar e estabelecer pontos de acesso dos elementos que compõem a descrição bibliográfica. 
Diversos países adotaram parte deste formato, no entanto, no início de 1999 a Library of Congress - Biblioteca do Congresso dos Estados Unidos e a National Library of Canada - Biblioteca Nacional do Canadá resolveram eliminar as diferenças existentes entre seus dois formatos USMARC e CAN/MARC, respectivamente, e publicam sob um novo nome, em edição única o MARC 21.

Na catalogação do documento utiliza-se regras para definir quais são as formas das entradas a serem descritas fisicamente; o próprio documento, os tesauros e as tabelas de classificação auxiliam na definição de seus assuntos. Já, o formato MARC organiza essas informações de forma a serem lidas pelo computador e possibilita a descrição bibliográfica de diferentes tipos de documentos (monografia, arquivo de computador, música, material cartográfico, imagens, software e outros).

O formato utiliza para tal a estrutura de campos fixos e variáveis, subcampos e indicadores. Cada registro MARC é dividido logicamente em campos. Há um campo para a informação de autor, um para título e assim por diante. Como a descrição de cada campo é muito extensa para ser definida dentro do registro, foram definidas tags (etiquetas) de três dígitos numéricos para identificar cada campo (FURRIE, 2003). No Quadro 2 estão descritos os campos básicos do formato MARC 21.

\begin{tabular}{ll} 
Campos & Descrição \\
\hline $0 X X$ & Informações de controle, números e códigos \\
$1 X X$ & Autoria (nome pessoal, entidade, evento) \\
$2 X X$ & Títulos, edição, imprenta \\
$3 X X$ & Descrição física \\
$4 X X$ & Série \\
$5 X X$ & Notas \\
\hline $6 X X$ & Entradas de assunto \\
$7 X X$ & Entradas secundárias (nome pessoal, entidade, evento, título) \\
$8 X X$ & Entradas secundárias de série
\end{tabular}




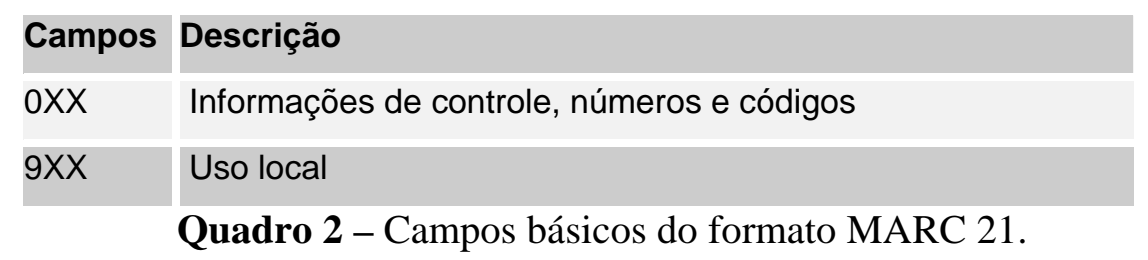

\section{CORRESPONDÊNCIA ENTRE DUBLIN CORE E FORMATO MARC 21}

Dados consistem de breves descrições padronizadas, seguindo elaborados códigos de regras. Cada padrão possui diferentes formas de especificação dos metadados utilizando diferentes linguagens de representação.

Todo elemento DC possui um valor. Cada elemento tem ocorrência ilimitada, e para diferenciar o valor de cada ocorrência, são utilizados qualificadores, os quais podem ter um identificador, chamado esquema e/ou modificador. Ambos servem para informar aos usuários e programas como interpretar o valor naquela ocorrência do elemento (SOUZA et al., 2002). Esses qualificadores e modificadores permitem ampliar o grau de especificidade dos dados a serem descritos, contribuindo para melhoria da satisfação do usuário.

O formato MARC 21 utiliza campos fixos de controle que contém informações codificadas usadas no processamento dos registros. Outras informações codificadas fazem parte de listas designadas pelo próprio formato, como códigos para Idiomas, País de publicação e Área geográfica. Todos os dados contidos nos campos, a partir do campo 010, se subdividem em subcampos, os quais são precedidos por um delimitador e um código de subcampo. O delimitador é formado por um símbolo (aqui representado pela barra invertida “”) que separa os diferentes subcampos. Os indicadores complementam esses dados e imprimem 'valor' ao conteúdo dos campos. Indicadores são números de um dígito e aparecem em duas posições logo após o número do campo.

Tomando por base essas observações foi possível identificar as correspondências existentes entre o formato MARC 21 e o padrão Dublin Core, apresentadas no Quadro 3. A 
extensibilidade, uma das principais características do padrão Dublin Core permite sua adaptação às necessidades adicionais de descrição. Na Agência de Informação Embrapa o uso de metadados DC foi consolidado, decidindo-se, porém, pela implementação de seus elementos e atributos, explicitados a seguir no Quadro 3 - Correspondência do padrão Dublin Core da Agência de Informação Embrapa e o Formato MARC 21:

\begin{tabular}{|c|c|}
\hline Dublin Core - Elementos & Formato MARC21 - Campos / Subcampos / Indicadores \\
\hline $\begin{array}{l}\text { Título } \\
\text { Modificador: Principal }\end{array}$ & 245 - Indicação de título - Subcampos la, lb, lh, In, Ip \\
\hline \multirow[t]{2}{*}{ Título: Principal - Idioma } & $\begin{array}{l}\text { Campo fixo } 008 \text { posição 35-37* } \\
041 \text { la - Código do Idioma* } \\
546 \text { la - Idioma - Informação textual na língua do material descrito }\end{array}$ \\
\hline & Nota: ${ }^{\star}$ dados codificados da tabela MARC-Code List for Languages \\
\hline $\begin{array}{l}\text { Título } \\
\text { Modificador: Alternativo }\end{array}$ & $\begin{array}{l}130 \text { - Entrada principal - Título uniforme - Subcampos la, In, Ip } \\
210 \text { la - Título abreviado } \\
240 \text { la - Título uniforme original } \\
246 \text { la - Forma variante do título } \\
740 \text { - Título adicional - Subcampos la, In, Ip }\end{array}$ \\
\hline Título: Alternativo - Idioma & $\begin{array}{l}130 V \text { - Entrada principal - Título uniforme - Idioma } \\
240 V \text { - Título uniforme - Idioma }\end{array}$ \\
\hline $\begin{array}{l}\text { Título } \\
\text { Modificador: Traduzido }\end{array}$ & 242 la - Título traduzido pela Agência de Catalogação \\
\hline \multirow[t]{2}{*}{ Título: Traduzido - Idioma } & $\begin{array}{l}242 \text { ly - Idioma } \\
041 \text { lh - Idioma - código do idioma do original e/ou tradução do texto } \\
\text { intermediário }\end{array}$ \\
\hline & Nota: dados codificados da tabela MARC-Code List for Languages \\
\hline $\begin{array}{l}\text { Título } \\
\text { Modificador: Série }\end{array}$ & $\begin{array}{l}440 \text { - Indicação de série - Subcampos la, In, Ip, Iv } \\
490 \text { - Indicação de série - Subcampos la, Iv }\end{array}$ \\
\hline Título: Série - Idioma & Não há correspondência \\
\hline $\begin{array}{l}\text { Criador } \\
\text { Modificador: Nome Pessoal }\end{array}$ & $\begin{array}{l}100 \text { - Entrada principal } \\
700 \text { - Entrada adicional } \\
100 \text { e } 700 \text { - Subcampos la, Ib, Ic, le, Iq } \\
720 \text { - Entrada adicional - Subcampos la, le }\end{array}$ \\
\hline Criador Endereço Pessoal & Não há correspondência \\
\hline $\begin{array}{l}\text { Criador } \\
\text { Modificador: Nome Corporativo }\end{array}$ & $\begin{array}{l}110 \text { - Entrada principal } \\
710 \text { - Entrada adicional } \\
110 \text { e } 710 \text { - Subcampos } \backslash a, I b, I n, I p \\
111 \text { - Entrada principal } \\
711 \text { - Entrada adicional } \\
111 \text { e } 711 \text { - Subcampos } \backslash a, \text { Ic, Id, Ie, In, Ip } \\
720 \text { - Entrada adicional - Subcampos la, le }\end{array}$ \\
\hline $\begin{array}{l}\text { Criador } \\
\text { Modificador: Endereço } \\
\text { Corporativo }\end{array}$ & $\begin{array}{l}110 \text { - Entrada principal } \\
710 \text { - Entrada adicional } \\
110 \text { e } 710 \text { - Subcampos la lb lu } \\
111 \text { - Entrada principal } \\
711 \text { - Entrada adicional } \\
111 \text { e } 711 \text { - Subcampos la le lu }\end{array}$ \\
\hline $\begin{array}{l}\text { Criador } \\
\text { Modificador: Afiliação }\end{array}$ & $\begin{array}{l}100 \text { lu - Entrada principal - Nome Pessoal - Afiliação } \\
700 \text { lu - Entrada adicional - Nome Pessoal - Afiliação }\end{array}$ \\
\hline
\end{tabular}


ARTIGO

\begin{tabular}{|c|c|}
\hline $\begin{array}{l}\text { Descrição } \\
\text { Modificador: Texto Livre }\end{array}$ & $\begin{array}{l}500 \text { la - Notas gerais } \\
501 \text { la - Notas Com } \\
502 \text { la - Nota de dissertação } \\
520 \text { la - Sumários, etc. } \\
\text { lb Indicador } 1 \text { = \# e } 2 \text { - Expansão da nota de sumário }\end{array}$ \\
\hline Descrição: Texto Livre - Idioma & Não há correspondência \\
\hline $\begin{array}{l}\text { Descrição } \\
\text { Modificador: Sumário }\end{array}$ & $\begin{array}{l}505 \text { - Notas de conteúdo - Subcampos la, Ig, It } \\
520 \text { la - Sumários, etc. } \\
\text { lb Indicador } 1 \text { = \# e } 2 \text { - Expansão da nota de sumário }\end{array}$ \\
\hline \multirow[t]{2}{*}{ Descrição: Sumário - Idioma } & 041 If - Idioma - código do idioma do conteúdo \\
\hline & Nota: dados codificados da tabela MARC-Code List for Languages \\
\hline $\begin{array}{l}\text { Descrição } \\
\text { Modificador: Resumo }\end{array}$ & $\begin{array}{l}520 \text { la - Sumários, etc. } \\
\text { lb Ind1=3 - Expansão da nota de resumo }\end{array}$ \\
\hline \multirow[t]{2}{*}{ Descrição: Resumo - Idioma } & 041 lb - Idioma - código do idioma do conteúdo \\
\hline & Nota: dados codificados da tabela MARC-Code List for Languages \\
\hline $\begin{array}{l}\text { Assunto } \\
\text { Modificador: Palavra-chave }\end{array}$ & $\begin{array}{l}650-\text { Assunto tópico } \\
\text { la Indicador } 2=\varnothing-\text { Assunto LCSH } \\
\text { la Indicador } 2=3-\text { Assunto NAL } \\
\text { la Indicador } 2=4-\text { Assunto }- \text { Fonte não especificada } \\
\text { lb - Termo tópico seguido de nome geográfico } \\
\text { lx - Subdivisão geral } \\
653 \text { la - Assunto tópico - Termo livre }\end{array}$ \\
\hline Assunto Palavra-chave - Idioma & Não há correspondência \\
\hline \multirow{2}{*}{$\begin{array}{l}\text { Assunto } \\
\text { Modificador: Categoria }\end{array}$} & $\begin{array}{l}050 \text { la - Número da LCCN (notação) } \\
080 \text { la - Número de classificação da CDU } \\
082 \text { la - Número de classificação da CDD }\end{array}$ \\
\hline & $\begin{array}{l}\text { Nota: notações e/ou números procedentes de tabelas de classificação } \\
\text { correspondentes. }\end{array}$ \\
\hline Assunto: Categoria - Idioma & Não há correspondência \\
\hline \multirow[t]{2}{*}{$\begin{array}{l}\text { Cobertura } \\
\text { Modificador: Temporal }\end{array}$} & $\begin{array}{l}045 \text { la - Código do período* } \\
650 \text { ly - Assunto tópico - subdivisão cronológica } \\
651 \text { ly - Assunto Nome Geográfico - subdivisão cronológica }\end{array}$ \\
\hline & Nota: *dados codificados da tabela Time Period Code Table \\
\hline Cobertura: Temporal - Idioma & Não há correspondência \\
\hline \multirow[t]{2}{*}{$\begin{array}{l}\text { Cobertura } \\
\text { Modificador: Espacial }\end{array}$} & $\begin{array}{l}043 \text { la - Código área geográfica* } \\
043 \text { lc - Código área geográfica "ISO 3166" } \\
522 \text { la - Nota geográfica da cobertura } \\
650 \text { lz - Assunto tópico - subdivisão geográfica } \\
651 \text { la - Assunto Nome Geográfico } \\
752 \text { - Entrada adicional - Nome de lugar hierárquico - Subcampos la, Id } \\
\text { (país ou entidade maior, cidade) }\end{array}$ \\
\hline & Nota:*dados codificados da tabela MARC Code List for Languages \\
\hline Cobertura: Espacial - Idioma & Não há correspondência \\
\hline $\begin{array}{l}\text { Publicador } \\
\text { Modificador: Nome Pessoal e/ou } \\
\text { Nome Corporativo }\end{array}$ & $260 \mathrm{lb}$ - Nome do Publicador \\
\hline $\begin{array}{l}\text { Publicador } \\
\text { Modificador: Endereço Pessoal e } \\
\text { Corporativo }\end{array}$ & 260 la - Lugar de Publicação \\
\hline $\begin{array}{l}\text { Publicador } \\
\text { Modificador: Afiliação }\end{array}$ & Não há correspondência \\
\hline
\end{tabular}




\section{Colaborador}

Modificador: Nome Pessoal e

Nome Corporativo

Endereço Pessoal e

Endereço Corporativo

Afiliação

Tipo de Recurso: Texto,

Imagem, Som, Dados, Software,

Interativo, Evento, Objeto Físico,

Coleção, Modelo, Lugar,

Participante, Serviço, Outros

\section{Identificador}

Esquemas: URI, ISBN, ISSN, FPI

Datas

Data de criação

Data da Última Modificação

Data Aproximada

\section{Formato}

Formato - Idioma

Fonte

Esquemas: Texto Livre, URI, ISBN, ISSN, FPI

Fonte - Idioma

Relação com outros recursos Modificador: Parte de

Esquemas: ISSN, ISBN, URI, FPI

Relação com outros recursos Modificador: Parte de - Idioma

Relação com outros recursos Modificador: Possui Parte Esquemas: ISSN, ISBN, URI, FPI Possui Parte - Idioma

Relação com outros recursos Modificador: Versão de Esquemas: ISSN, ISBN, URI, FPI

Versão de - Idioma

Relação com outros recursos Modificador: Possui versão
Não há correspondência

Campo fixo de controle 006 posição 00-17

Campo fixo de descrição física 007

Campo fixo de controle 008 posição 18-34

Nota: Dados codificados

020 la - ISBN

022 la - ISSN

520 lu - Sumários, etc. - URI

856 lu - Ind1=4 Ind2=2 - Acesso e localização eletrônica - URI

260 IC - Data de publicação

$260 \mathrm{lg}$ - Data de manufatura

533 ld - Data de reprodução

Campo fixo de controle 008 posição 07-10

Nota: No campo 260 subcampo Ic pode conter data de publicação e/ou data de copyright.

A posição 06 do campo fixo 008 complementa as informações da posição 07-10, informa se a data é aproximada, copyright, reimpressão, etc.

300 - Descrição física - Subcampos la, Ic

340 - Meio (suporte) físico - Subcampos la, lb, le, li

533 le - Descrição física da reprodução

856 lq - Ind1=4 Ind2=2 - Tipo de formato eletrônico

Não há correspondência

786 - Entrada 'original' dos dados - Subcampos la, lb, Ic, Id, lh, lk, It, lo, $\mathrm{lz}, \mathrm{Ix}$

Não há correspondência

440 - Indicação de série - Entrada adicional - Subcampos la, In, Ip, Iv, Ix 490 - Indicação de série - Subcampos la, IV, Ix

760 - Entrada de série - Subcampos la, lb, lc, ld, Ig, lh, It, lo, Ix

773 - "Host Item Entry" - Subcampos la, lb, Id, Ig, lh, It, lo, Ix, Iz

800 - Entrada adicional para série - Subcampos la, If, In, Ip, It, IV

810 - Entrada adicional para série - Subcampos la, Ib, Ic, If, In, Ip, It, IV

811 - Entrada para série - Subcampos la, Ic, Id, le, If, In, Ip, It, IV

830 - Entrada adicional para série - Subcampos la, If, In, Ip, It, IV

$800 \mathrm{~V}$ - Entrada adicional para série - Nome pessoal - Idioma

$810 \mathrm{~V}$ - Entrada adicional para série - Nome corporativo - Idioma

$811 \mathrm{~V}$ - Entrada adicional para série - Nome do evento - Idioma

$830 \mathrm{~V}$ - Entrada adicional para série - Título uniforme - Idioma

774 - "Constituent Unit Entry" - Subcampos la lb Ic Id Ih In It lo Ix Iz

\section{Não há correspondência}

775 - Entrada para outras edições - Subcampos la lb lc ld lg lh In It lo Ix I

786 - Entrada 'original' dos dados la lb lc ld lh lk In It lo Ix Iz

775 le - Entrada para outras edições - Idioma (informações codificadas)

Nota: dados codificados da tabela MARC Code List for Languages

775 - Entrada para outras edicões - Subcampos la lb Ic ld la lh In It lo Ix 
Esquemas: ISSN, ISBN, URI, FPI Iz

Possui versão - Idioma

Relação com outros recursos

Modificador: Formato de

Esquemas: ISSN, ISBN, URI, FPI

Formato de - Idioma

Relação com outros recursos

Modificador: Possui formato

Esquemas: ISSN, ISBN, URI, FPI

Possui formato - Idioma

Relação com outros recursos

Modificador: Referências

Esquemas: ISSN, ISBN, URI, FPI

Referências - Idioma

Relação com outros recursos

Modificador: Referenciado por

Esquemas: ISSN, ISBN, URI, FPI

Referenciado por - Idioma

Relação com outros recursos

Modificador: Baseado em

Esquemas: ISSN, ISBN, URI, FPI

Baseado em - Idioma

Relação com outros recursos

Modificador: Base para

Esquemas: ISSN, ISBN, URI, FPI

Base para - Idioma

Relação com outros recursos

Modificador: Requer

Esquemas: ISSN, ISBN, URI, FPI

Requer - Idioma

Relação com outros recursos

Modificador: Requerido por

Esquemas: ISSN, ISBN, URI, FPI

Requerido por - Idioma

Relação com outros recursos

Modificador: Continua em

Esquemas: ISSN, ISBN, URI, FPI

Continua em - Idioma

Relação com outros recursos

Modificador: Continuação de

Esquemas: ISSN, ISBN, URI, FPI

Continuação de - Idioma

Direitos autorais

Esquemas: Texto livre, URI

Direitos autorais - Idioma

Quadro 3 - Correspondência do padrão Dublin Core da Agência de Informação Embrapa e

775 le - Entrada para outras edições - Idioma (informações codificadas)

Nota: dados codificados da tabela MARC Code List for Languages

530 - Nota sobre formato físico adicional disponível - Subcampos la lb lc lu

776 - Entrada adicional para formato físico - Subcampos la lb lc ld lg lh In It lo Ix Iz

Não há correspondência

530 - Nota sobre formato físico adicional disponível - Subcampos la lb lc lu

776 - Entrada adicional para formato físico - Subcampos la lb lc ld lg lh In It lo Ix Iz

Não há correspondência

Não há correspondência

Não há correspondência

510 - Notas de citação/referências - Subcampos la lb lc lx

Não há correspondência

Não há correspondência

Não há correspondência

Não há correspondência

Não há correspondência

538 - Notas sobre detalhes do sistema - Subcampos la lu

Não há correspondência

Não há correspondência

Não há correspondência

785 - Entrada posterior - Subcampos la lb lc ld lg lh In It lo Ix Iz

Não há correspondência

780 - Entrada anterior - Subcampos la lb lc ld lg Ih In It lo Ix Iz

Não há correspondência

506 - Notas de restrição de acesso - Subcampos la lu

540 - Termos de uso e reprodução - Subcampos la lu

Não há correspondência

o Formato MARC 21 


\section{RESULTADOS E DISCUSSÃO}

Este estudo apresenta as características do padrão de metadados Dublin Core na Agência de Informação Embrapa e do formato MARC 21, bem como seus campos, subcampos e elementos correspondentes, visando principalmente sua utilização para subsidiar o desenvolvimento de ferramentas que permitam a conversão e a integração de dados.

Além da análise de correspondência entre ambos (MARC 21 e DC), este estudo contribui de forma efetiva para os esforços de integração e de interoperabilidade, aplicadas à Agência de Informação Embrapa, em relação a qualquer sistema que adota o MARC 21 como formato de entrada de dados.

A partir deste estudo é possível constatar que, de uma forma geral o padrão Dublin Core destaca-se pela sua simplicidade e interoperabilidade, uma vez que o mesmo apresenta um conjunto de elementos capazes de abstrair informações importantes dos objetos tais como: imagens, sons e vídeos. Essas características facilitam o tratamento das informações, facilitando, igualmente sua busca e recuperação.

De acordo com Gonçalves (2005), ao contrário do Dublin Core que possui um número pequeno de atributos com o objetivo de ser de fácil utilização, o formato MARC possui um número bem maior de atributos, pois seu objetivo é ser mais completo. Apesar das diferenças e da complexidade de descrição dos metadados no formato MARC 21, podese constatar que o conjunto de descritores deve conter apenas informações apropriadas e suficientes para descrever os dados de forma que a informação nele contida além de ser compreendida por qualquer pessoa, possa também ser compilada e/ou interpretada pelas novas tecnologias para tratamento da informação, pois deve servir de subsídio a sistemas de buscas e recuperação de informações. 
Cabe à instituição, que tem como política o compartilhamento de dados, definir quais campos serão utilizados ou reaproveitados e fazer a customização das ferramentas de catalogação, de acordo com as necessidades do seu sistema. Como cada um dos padrões possui comunidades e instituições que os utilizam para distribuição, uma abordagem ideal do problema, que é o princípio básico de integração de dados, seria dispor de mecanismos que permitam abstrair o tipo de representação documental, integrando-os numa mesma aplicação (XAVIER, 2000).

\section{CONSIDERAÇÕES}

A integração e o compartilhamento de dados entre sistemas, bem como a interoperabilidade entre aplicações, além de desejáveis são viáveis, notadamente em relação a sistemas que utilizam o padrão de metadados Dublin Core e o formato MARC 21, para descrição de dados.

Considera-se, pois, necessário o compartilhamento de metadados, que venham contribuir para o sucesso da interoperabilidade que está, eminentemente, relacionado à organização, tanto dos recursos de informação, quanto dos seus metadados, disponíveis em bases de dados, em redes internas (intranets) ou externas (internet).

Um fator importante a considerar refere-se à qualidade dos dados gerados a partir de padrões de consenso internacional, que segundo Gonçalves (2005) inclui os aspectos de precisão, fidelidade, critérios de seleção, generalizações, consistência dos dados, definições utilizadas e metadados sobre as fontes de dados. Nesse sentido, pode-se citar, ainda, a adoção de padrões de representação descritiva dos recursos de informação - a catalogação, propriamente dita. O uso efetivo das regras de descrição visa enriquecer as representações dos recursos e facilitar o compartilhamento desses dados, de modo que os usuários finais sejam plenamente satisfeitos quanto à qualidade da informação recuperada.

Como vantagem ter-se-á a dinamização do processo de catalogação de recursos de informação e a eliminação de esforços repetitivos e redundantes, suprimindo com isso o 
retrabalho de tratamento da informação, além de contribuir para a integração e compartilhamento de dados e interoperabilidade entre sistemas, como o sistema Agência.

\section{REFERÊNCIAS}

BUTTÀ, B. Conversione tra Marc 21 e Dublin Core. In: Buttà, Basilio. Metadati negli open archive: tecniche di conversione dei formati dei record nel formato MARC21 utilizzato da CDSware. Italy: University of Messina, 2004. cap. 5. Thesis of degree, Corso di Laurea in Informatica, University of Messina (Italy). Disponível em: <http://eprints.rclis.org/archive/00003022/06/cap_5.PDF>. Acesso em: 08. jun. 2006.

CHAVES, M. S. Padrões em bibliotecas digitais. Porto Alegre: PUC-RS, 2002. 54 p. Disponível em: < http://xldb.di.fc.ul.pt/ mohaves/pg_portugues/master/trabalhos/ti1.pdf>. Acesso em: 10 nov. 2005.

COMITÊ EXECUTIVO DE GOVERNO ELETRÔNICO. e-PING padrões de interoperabilidade de governo eletrônico - documento de referência versão 1.5: [S.l.], 2005. 66 p. Disponível em: $<$ http://www.governoeletronico.gov.br/governoeletronico/publicacao/down_anexo.wsp?tm p.arquivo=E15_677e-PING\%20v1.5\%2016\%2012\%2020051.pdf>. Acesso em: 24 jul. 2006.

\section{DUBLIN CORE METADATA INITIATIVE. Dublin Core metadata element set, version} 1.1: reference description. [S.l.], 2004. Disponível em: <http://dublincore.org/documents/dces/>. Acesso em: 23 fev. 2006.

FURRIE, B. Understanding MARC bibliographic: machine-readable cataloging. 7th. ed. rev. Washington, D. C.: Library of Congress; Follet Software, 2003. Disponível em: <http://www.loc.gov/marc/umb/>. Acesso em: 27 fev. 2006. 
GONÇALVES, J. L. Estudo de gestão de dados não convencionais baseada em metadados para o ambiente de dispositivos móveis. 2005. 78 p. Dissertação (Mestrado) Informática, Universidade Federal do Paraná, Curitiba. Disponível em: <http://dspace.c3sl.ufpr.br/dspace/bitstream/1884/3246/1/Disserta\%3F\%3Fo_Juliano.pdf>. Acesso em: 08 jun. 2006.

LIBRARY OF CONGRESS. Network Development and MARC Standards Office. Dublin Core/MARC/GILS Crosswalk. Disponível em: < http://www.loc.gov/marc/dccross.html> Acesso em: 18 maio 2006.

LIBRARY OF CONGRESS. Network Development and MARC Standards Office. MARC

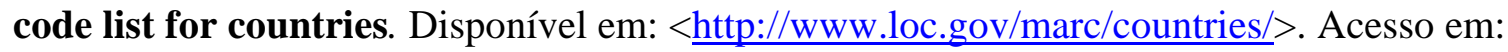
18 maio 2006.

LIBRARY OF CONGRESS. Network Development and MARC Standards Office. MARC code list for geographic areas. Disponível em: < $\underline{\text { http://www.loc.gov/marc/geoareas/>. }}$ Acesso em: 18 maio 2006.

LIBRARY OF CONGRESS. Network Development and MARC Standards Office. MARC code list for languages. Disponível em: < http://www.loc.gov/marc/languages/>. Acesso em: 18 maio 2006.

LIBRARY OF CONGRESS. Network Development and MARC Standards Office. MARC21 concise format of bibliographic data. Disponível em: <http://www.loc.gov/marc/bibliographic/ecbdhome.html>. Acesso em: 18 maio 2006.

LIBRARY OF CONGRESS. Network Development and MARC Standards Office. MARC to Dublin Core crosswalk. Disponível em: <http://www.loc.gov/marc/marc2dc.html> Acesso em: 18 maio 2006. 
PEREIRA, A. M.; RIBEIRO JÚNIOR, D. I.; NEVES, G. L. C. Metadados para a descrição de recursos da Internet: as novas tecnologias desenvolvidas para o padrão Dublin Core e sua utilização. Revista ACB: Biblioteconomia em Santa Catarina, Florianópolis, v. 10, n. $1, \quad$ p. $8-39, \quad$ jan./dez. $2005 . \quad$ Disponível em: $<$ http://www.acbsc.org.br/revista/ojs/viewarticle.php?id=113\&layout=abstract $>$. Acesso em: 06 jun. 2006.

RELVÃO, R. R. Elementos de metadados para a aprendizagem à distância. Guimarães: Universidade do Minho, 2003. 46 p. Disponível em: $<$ http://papadocs.dsi.uminho.pt:8080/retrieve/285/Elementos+de+Metadados+para+a+Apre ndizagem+\%C3\%A0+Dist\%C3\%A2ncia.doc>. Acesso em: 08 jun. 2006.

ROSETTO, M.; NOGUEIRA, A. H. Aplicação de elementos metadados Dublin Core para descrição de dados bibliográficos on-line da biblioteca digital de teses da USP. In: SEMINÁRIO NACIONAL DE BIBLIOTECAS UNIVERSITÁRIAS, 12., 2002, Recife.

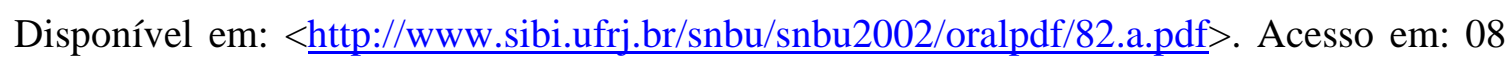
jun. 2006.

SOUZA, M. I. F.; ALVES, M. das D. R.; SANTOS, A. D. dos; CINTRA, M. A. M. de U.; OLIVEIRA, M. J. de. Guia para descrição de recurso eletrônico no Sistema Agência utilizando Dublin Core. Campinas: Embrapa Informática Agropecuária, 2002. 44 p. (Documentos, 11). Disponível em: $<$ http://www.cnptia.embrapa.br/modules/tinycontent3/content/2002/doc11.pdf $>$. Acesso em: 20 set. 2005.

SOUZA, M. I. F.; MOURA, M. F.; SANTOS, A. D. dos. Estudo comparativo entre os metadados da Agência de Informação Embrapa e do Acervo Documental do AINFO. Campinas: Embrapa Informática Agropecuária, 2005. 10 p. (Comunicado Técnico, 66). Disponível em: 
<http://www.cnptia.embrapa.br/modules/tinycontent3/content/2004/comtec66.pdf $>$. Acesso em: 23 out. 2005.

SOUZA, M. I. F.; VENDRUSCULO, L. G.; MELO, G. C. Metadados para a descrição de recursos de informação eletrônica: utilização do padrão Dublin Core. Ciência da Informação, v. 29, n. 1, p. 93-102, abr. 2000. . Disponível em: <http://www.scielo.br/pdf/ci/v29n1/v29n1a10.pdf>. Acesso em: 27 set. 2005.

XAVIER, H. A. F. Padrões de metadados para bibliotecas digitais. Belo Horizonte: UFMG, $2005 . \quad 38$ p. Disponível em: <http://www.npdi.dcc.ufmg.br/orientacoes/especializacao/hamilton/Monografia\%20UFMG \%202005_Hamilton\%20Xavier.pdf>. Acesso em: 08 jun. 2006. 


\section{Maria das Dores Rosa Alves}

Bibliotecária. Especialista em Sistemas Automatizados de Informação Científica e Tecnológica. Consultora em Organização e Tratamento da Informação da Embrapa Informática Agropecuária. maria@cnptia.embrapa.br

\section{Marcia lzabel Fugisawa Souza}

Mestre em Biblioteconomia. Técnico em Nível Superior da Embrapa Informática Agropecuária. marcia@cnptia.embrapa.br 